\title{
Neural response to evaluating depression predicts perceivers' mental health treatment recommendations
}

\author{
Anne C. Krendl $^{1}$ • Brittany S. Cassidy ${ }^{1}$
}

Published online: 11 September 2017

(C) Psychonomic Society, Inc. 2017

\begin{abstract}
Nonstigmatized perceivers' initial evaluations of stigmatized individuals have profound consequences for the well-being of those stigmatized individuals. However, the mechanism by which this occurs remains underexplored. Specifically, what beliefs about the stigmatized condition (stigma-related beliefs) shape how nonstigmatized perceivers evaluate and behave toward stigmatized individuals? We examined these questions with respect to depression-related stigmatization because depression is highly stigmatized and nondepressed individuals' behavior (e.g., willingness to recommend treatment) directly relates to removing stigma and increasing well-being. In Study 1, we identified common stigma-related beliefs associated with depression (e.g., not a serious illness, controllable, threatening), and found that only perceptions that depression is a serious condition predicted nondepressed perceivers' willingness to recommend mental health treatment. Moreover, perceivers' beliefs that depression is a distressing condition mediated the relationship between perceived seriousness and treatment recommendations (Study 1). In Study 2, we used fMRI to disentangle the potential processes connecting distress to nondepressed perceivers' self-reported treatment intentions. Heightened activity in the dorsomedial prefrontal cortex (dmPFC) - a region widely implicated in evaluating others - and the
\end{abstract}

Electronic supplementary material The online version of this article (https://doi.org/10.3758/s13415-017-0534-8) contains supplementary material, which is available to authorized users.

Anne C. Krendl

akrendl@indiana.edu

1 Department of Psychological and Brain Sciences, Indiana University, 1101 E. 10th St., Bloomington, IN 47405, USA ventrolateral prefrontal cortex (vlPFC) - a region widely implicated in regulating negative emotions - emerged when nondepressed perceivers evaluated individuals who were ostensibly depressed. Beliefs that depression is a distressing condition mediated the relationship between dmPFC (but not vlPFC) activity and nondepressed individuals' selfreported treatment recommendations.

Keywords Stigma - Dorsomedial prefrontal cortex · fMRI · Depression

In everyday life, we constantly evaluate the people we encounter, often without conscious awareness (Bargh \& Pietromonaco, 1982; Uleman, Newman, \& Moscowitz, 1996). Perceivers' initial evaluations of others predict their subsequent behavior toward their targets (e.g., Ambady \& Rosenthal, 1992) and powerfully impact their targets' wellbeing (Brewer, 1988; Fiske \& Neuberg, 1990). The dorsomedial prefrontal cortex [dmPFC] has been widely implicated in evaluating others (for meta-analyses and reviews, see Denny, Kober, Wager, \& Ochsner, 2012; Mitchell, 2009; Van Overwalle, 2009). However, the extent to which activation in the $\mathrm{dmPFC}$ and related regions (e.g., ventrolateral prefrontal cortex, temporo-parietal junction, amygdala, and posterior cingulate cortex; Cloutier, Gabrieli, O'Young, \& Ambady, 2011; Mende-Siedlecki, Cai, \& Todorov, 2012; Schiller, Freeman, Mitchell, Uleman, \& Phelps, 2009) predict behavior toward stigmatized individuals outside of the scanner remains largely underexplored (but see Richeson et al., 2003).

Stigma, which refers to any attribute that reduces someone "from a whole and usual person to a tainted, discounted one" (Goffman, 1963:3), has highly pernicious outcomes for its targets (Major \& O’Brien, 2005). Social stigma is detected rapidly (Krendl, Zucker, \& Kensinger, 2016). Moreover, when 
nonstigmatized perceivers evaluate highly stigmatized individuals (e.g., homeless individuals), they engage disparate patterns of neural activation (e.g., reduced $\mathrm{dmPFC}$ activation) than when they evaluate other stigmatized individuals or nonstigmatized controls (e.g., Harris \& Fiske, 2006; Krendl, Macrae, Kelley, Fugelsang, \& Heatherton, 2006). Because the dmPFC plays a central role in evaluating others (Denny et al., 2012), reduced activation in this region for some stigmatized targets may shed light on why these groups are dehumanized (e.g., Fiske, Cuddy, Glick, \& Xu, 2002).

However, it remains largely unknown how nonstigmatized perceivers' initial evaluations of stigmatized individuals might affect behavior toward those individuals. This question is particularly relevant for mental health conditions such as depression. Mental illness is one of the most stigmatized conditions a person can have (e.g., Hinshaw, 2006) and is associated with robust and pervasive bias (e.g., for review, see Corrigan, 2004). Depression is the most common mental illness in the U.S., affecting nearly 15 million Americans each year (National Institute of Mental Health, 2012). However, as many as two thirds of people suffering from depression do not seek treatment (National Alliance on Mental Illness, 2001), and concerns with being stigmatized have been widely cited as one of the most prominent reasons why (for review, see Corrigan, 2004). Untreated depression is the leading cause of disability in the U.S. (Greenberg, et al., 2003) and the primary cause of suicide, which is the 10th leading cause of death in the U.S. (Centers for Disease Control and Prevention, 2013). Ironically, treatment directly relates to removing these individuals' stigma and increasing their well-being (for review, see Corrigan, 2004).

Although some of the causes and consequences of mental health stigma have been characterized (e.g., Link \& Phelan, 2006; Pescosolido, Martin, Lang, \& Olafsdottir, 2008), how depression-related stigma reduces treatment recommendations more broadly remains unclear. One possibility is that depression-related stigma may affect perceivers' treatment recommendations by activating common beliefs about the stigmatized condition, including the extent to which it is perceived to be controllable, dangerous, not serious, or unfamiliar (Deaux, Reid, Mizrahi, \& Ethier, 1995; Fiske et al., 2002; Goffman, 1963; Major \& O’Brien, 2005). These beliefs may then generalize to beliefs about the affective impact of the condition (e.g., it is distressing, it is pitiable) (e.g., Batson, Fultz, \& Schoenrade, 1987; Leventhal et al., 1992). For instance, if perceivers believe that depression is a serious condition, they may deem the condition to be more distressing (e.g., Leventhal et al., 1992) and thus be more willing to recommend mental treatment.

Because both depressed and nondepressed individuals do not differ in their levels of explicit or implicit mental health stigma (Teachman, Wilson, \& Komarovskaya, 2006), it is likely that these stigmas stem from negative societal beliefs about depression. Thus, understanding how nondepressed perceivers' stigma toward depression affects their willingness to recommend treatment is an important first step in developing interventions that are effective in reducing stigma and promoting treatment. If, as predicted, stigma influences perceivers' beliefs about the affective impact of depression, this could reduce nondepressed perceivers' willingness to recommend mental health treatment either by affecting how they initially evaluate depressed individuals (e.g., Batson et al., 1987; Harris \& Fiske, 2006) or by decreasing the regulatory effort they engage to override their negative bias (e.g., Devine, 1989). Simply put, if either perceivers' initial evaluations or regulatory effort predict their treatment recommendations, their beliefs about the affective impact of depression (e.g., how distressing it is) may mediate that relationship.

A neuroimaging approach may be uniquely beneficial in disentangling these disparate mechanisms because the neural correlates underlying evaluating others (e.g., Denny et al., 2012; Mitchell, 2009; Van Overwalle, 2009) and regulating negative emotions (Ochsner, Silvers, \& Buhle, 2012) have been well characterized. The present work is the first to examine the potential neurobiological mechanism by which neural activity while evaluating stigmatized (e.g., depressed) individuals generalizes toward endorsing treatment. The current work is an important first step toward developing interventions that might reduce the stigma associated with mental health treatment. For instance, if reduced regulatory effort in response to evaluating depressed individuals predicts' nondepressed perceivers' reduced willingness to recommend mental health treatment, it would suggest that interventions that promote regulatory effort might be the most effective in reducing the stigma associated with seeking mental health treatment.

In Study 1, we sought to determine how perceivers' stigmarelated beliefs about depression influenced their willingness to recommend treatment (which is unknown). We predicted that the stigma-related beliefs that affected perceivers' treatment recommendations would be mediated by their related beliefs about the affective impact of depression. Study 2 then used fMRI to elucidate a potential neural mechanism by which this may occur. We anticipated that nondepressed perceivers' initial evaluations of depressed individuals or their regulatory effort during those evaluations would predict their willingness to recommend mental health treatment, but that this effect would be mediated by their beliefs about the affective impact of depression. Based on the literature, we hypothesized that this might happen in one of two ways. First, perceivers who believe that depression is a distressing condition may engage more dorsomedial prefrontal cortex (dmPFC) activity when they evaluate depressed individuals (suggesting stronger recruitment of impression formation processes). The extent of $\mathrm{dmPFC}$ engagement may then increase their willingness to recommend treatment. Second, perceivers who believe that depression is a distressing condition may have higher 
activation in neural regions associated with bias regulation (e.g., ventrolateral prefrontal cortex) when they evaluate depressed individuals. The extent of this engagement may, in turn, also increase their willingness to recommend treatment.

\section{Study 1}

A wide body of research has shown that the extent to which people recommend societal beliefs about a stigmatized condition, including the extent to which it is perceived to be controllable, dangerous, not serious, or unfamiliar (Deaux et al., 1995; Fiske et al., 2002; Goffman, 1963; Major \& O’Brien, 2005) influences their behaviors toward stigmatized individuals. Perceivers' stigma-related beliefs might then influence their beliefs about the affective impact of the stigmatized condition (e.g., Fiske et al., 2002). For instance, prior work suggests that recommending treatment for others requires perceiving the tobe-treated illness as being serious (e.g., Janz \& Becker, 1984), and believing that having the condition would be distressing (for review, see Leventhal et al., 1992). We thus predicted that perceivers' stigma-related beliefs toward depression (e.g., that depression is a serious condition) would predict their willingness to recommend treatment for depressed individuals, but that this relationship would be mediated by their beliefs about the affective impact of depression (e.g., how distressing they perceived having depression to be; how much sympathy they have for individuals suffering from depression).

Three important considerations might also impact perceivers' treatment recommendations: (1) individual differences in beliefs about seeking treatment more generally; (2) depression-related stigmatization (which has been widely shown to disrupt treatment seeking; Corrigan, 2004); and (3) personal experiences with depression (either by oneself or a close friend). Regarding individual differences in beliefs about seeking treatment, some nonstigmatized perceivers might be more willing to seek treatment for any illness, and/or more likely to perceive any condition as having a negative affective impact on its bearer. Our central interest in the current study was to identify the stigma-related beliefs that predicted nondepressed perceivers' willingness to recommend treatment for depression above and beyond individual differences in perceivers' general beliefs about all illnesses. We therefore also measured treatment recommendations and beliefs for a nonstigmatized illness: migraines. With respect to measuring personal experiences with depression, prior work suggests that perceivers' mental health stigma does not differ as a function of whether or not they personally know someone with a mental health problem (e.g., a family member; Crisp, Gelder, Rix, Meltzer \& Rowlands, 2000; Moses, 2010). However, it is less well understood whether familiarity affects mental health treatment recommendations. We therefore included this item as a conceptual replication and extension of prior work.

\section{Method}

\section{Participants}

Seventy Indiana University undergraduate students (18-30 years of age, 48 female) participated. Of the 70 participants, 55 identified as Caucasian (78.6\%), 7 (10\%) as Black, 3 (4\%) as Asian, $4(5.7 \%)$ as more than one race, and 1 did not provide race information. A priori power analyses conducted in G*Power (Faul, Erdfelder, Lang, \& Buchner, 2007) using a medium effect size $\left(f^{2}=.35\right)$, alpha $=.05$, and power $=.95$, indicated 67 participants would detect effects.

\section{Materials}

We measured treatment recommendations, sympathy, and distress using two novel vignettes describing two different individuals. One vignette described an individual suffering from symptoms commonly associated with depression, while the other described an individual suffering from migraines (which served as the control condition). Pilot testing with a separate group of undergraduates at Indiana University confirmed that migraines and depression were perceived as similarly familiar, treatable, and emotionally arousing (see Supplemental Materials for validation of these vignettes). The vignettes were written in similar styles and length, and the gender of the described individual always matched participant gender. Participants viewed the two vignettes in pseudorandom order, and were asked after reading each. "How distressing do you believe the person's condition is? How sympathetic are you to the person described?" and "If you were friends with this person, how likely would you be to recommend that s/he seek professional treatment?" For all items, the scale ranged from 1 (not at all) to 7 (very much).

Participants then completed a series of filler tasks. After, we measured their stigma-related beliefs about depression using the following ratings (presented in pseudorandom order): "How responsible do you think someone with depression/migraines is for their condition?" (responsibility); "How likely do you think it is that someone suffering from depression/migraines can change their condition?" (changeability); "How serious do you think depression/ migraines is?" (seriousness of disorder); "How much of a threat do you think people with depression/migraines pose to themselves or others?" (perceived threat). All ratings were made on a 1 (not at all) to 7 (very much) scale and were self-paced. Prior work has identified these beliefs as the primary dimensions by which stigmatized conditions are evaluated (e.g., Deaux et al., 1995; Feldman \& Crandall, 2007; Goffman, 1963). 
We measured participants' personal familiarity with depression by asking them to indicate whether they or someone they knew had ever suffered from depression or migraines (via yes/no response), and, if so, how well. They also completed the Internalized Stigmatization Scale (ISS; Ritsher, Otilingam, \& Grajales, 2003), which measures mental health stigmatization. We modified the ISS from its original version to refer specifically to depression instead of mental illness. The interitem reliability was acceptable (Cronbach's $\alpha=.72$ ).

\section{Results}

\section{Comparing ratings for depression and migraines}

Consistent with our pilot testing for the vignettes (see Supplemental Materials), participants did not differ in their willingness to recommend treatment for depression $\left(M_{\text {Depression }}=6.07, S D=1.29\right)$ and migraines $\left(M_{\text {Migraines }}=\right.$ $6.21, S D=1.41 ; t<1)$. They also did not differ in their perceptions that the two conditions were distressing $\left(M_{\text {Depression }}=\right.$ $\left.5.46, S D=1.26 ; M_{\text {Migraines }}=5.57, S D=1.03\right)$ or elicited sympathy $\left(M_{\text {Depression }}=5.54, S D=1.45 ; M_{\text {Migraines }}=5.69\right.$, $S D=1.22$ ), both $t \mathrm{~s}<1$. Regarding their stigma-related beliefs toward the two illnesses, perceivers did not differ in their perceptions regarding controllability of the two illnesses (responsibility: $M_{\text {Depression }}=2.61, S D=1.61 ; M_{\text {Migraines }}=2.27, S D=$ 1.60 ; changeability: $M_{\text {Depression }}=2.73, S D=1.31 ; M_{\text {Migraines }}=$ $2.70, S D=1.41)$, both $t \mathrm{~s}<1.64, p \mathrm{~s}>.10$. However, they rated depression as being more serious than migraines $\left(M_{\text {Depression }}=\right.$ $\left.6.29, S D=1.00 ; M_{\text {Migraines }}=5.37, S D=1.33\right) ; t(69)=5.63, p<$ .001 , as well as more threatening $\left(M_{\text {Depression }}=4.71, S D=\right.$ $\left.1.63 ; M_{\text {Migraines }}=2.41, S D=1.32\right) ; t(69)=11.52, p<.001$.

A closer examination of the ratings for the two illnesses revealed that they were all highly correlated with one another (all $r \mathrm{~s}>.27, p s<.05$ ). For instance, perceptions that migraines is a serious disorder was correlated with perceptions that depression is a serious disorder, $r(68)=.35, p=.003$. The same pattern emerged for perceptions that migraines and depression are threatening, $r(68)=.38, p<.001$, and distressing, $r(68)=$ $.53, p<.001$. (See Supplemental Materials for a complete table of the correlations between all of the ratings.) Together, the fact that these ratings were highly correlated suggested that individual differences contributed to perceivers' ratings. That is, the extent to which an individual perceived depression to be a serious illness was likely influenced more generally by how serious the perceiver found any illness to be. To control for individual differences in responding, we therefore created difference scores for each rating (e.g., willingness to recommend treatment for depression-willingness to recommend treatment for migraines). Using these difference scores allowed us to ask the more specific question: why are perceivers' more (or less) likely to recommend treatment for depression than migraines?
Identifying the stigma-related beliefs that predicted mental health treatment recommendation

Using the abovementioned difference scores, we used linear regression to identify stigma-related belief(s) (low perceived seriousness, high responsibility, high changeability, and/or high threat) that predicted their treatment recommendations for depression (vs. migraines) when controlling for participants' familiarity (either themselves or someone they knew) with depression and their mental health stigma beliefs (using ISS). The overall model was significant, $F(6,68)=4.49, p=$ .001 , and accounted for $30 \%$ of the overall variance in treatment recommendations. ${ }^{1}$ Supporting our hypothesis, perceived seriousness for depression (vs. migraines) was the only significant predictor $(B=.40, t=3.658 p=.001)$. None of the other stigma-related beliefs (responsibility, changeability, or threat) predicted treatment recommendations (all $\beta \mathrm{s}<.21$, $t \mathrm{~s}$ $<1.80, p \mathrm{~s} \geq .08$ ). See Table 1 for all relevant statistics.

\section{Perceived seriousness mediates relationship between distress and treatment recommendations}

We next examined whether perceivers' beliefs that depression is a distressing condition or their sympathy for individuals suffering from depression (vs. migraines) were associated with perceived seriousness. The overall model was significant, $F(2,68)=10.32, p<.001$, and accounted for $24 \%$ of the overall variance. Distress was the only significant predictor $(\beta=.44, t=$ $3.68, p<.001$; sympathy: $\beta=.10, t=.81, p=.42){ }^{2}$ Consistent with our hypothesis, mediation analyses using PROCESS with 1,000 bootstrap samples (Hayes, 2012) showed that although perceptions that depression (vs. migraines) is a serious illness positively predicted treatment recommendations, it acted

\footnotetext{
${ }^{1}$ Participants who did not know anyone with depression (20 out of the 70 participants) were coded as zero for this analysis. However, excluding those 20 participants does not affect the overall model. Indeed, the model was still significant, $F(5,48)=6.28, p<.001$, for the 50 participants who knew someone with depression, and accounted for $42.2 \%$ of the overall variance. Again, the belief that depression is serious was significant in this model $(B=.46, t=$ $3.84, p<.001)$. Familiarity more generally did not predict treatment intentions alone or in the model (all $t s<1$ ). Indeed, the first model in Study 1 remained significant when excluding familiarity and bias (for all 70 participants), $F(4$, $69)=6.29, p<.001$, and accounted for $24 \%$ of the overall variance.

${ }^{2}$ When we ran the regressions from Study 1 with only White participants, the same pattern of results emerged. Specifically, the overall model predicting treatment recommendations from perceivers' stigma-related belief(s) toward depression (vs. migraines) (perceived seriousness, responsibility, changeability, and/or threat) when controlling for participants' familiarity with depression and their mental health stigma was significant, $F(6,54)=2.86, p=.028$, and accounted for $24 \%$ of the overall variance in treatment recommendations. As with the full sample, only perceived seriousness was significant $(\beta=.424, t=$ $3.31, p=.002$ ). We also found that the overall model with perceived distress and sympathy toward depression as potential predictors for beliefs that depression is a serious illness was significant, $F(2,54)=7.18, p=.002$, and accounted for $21.6 \%$ of the overall variance. As with the full sample, perceived distress was the only significant predictor $(B=.42, t=3.12, p=.003$; sympathy: $\beta=.11, t=.83, p=.41$ ).
} 
Table 1 Summary of regression predicting willingness to recommend mental health treatment

\begin{tabular}{lllll}
\hline Variable & $\beta$ & $t$ & $\begin{array}{l}R \\
.550\end{array}$ & $\begin{array}{c}R^{2} \\
.303\end{array}$ \\
\hline Internalized stigma & .116 & 1.076 & & \\
Personal Familiarity & .115 & 1.042 & & \\
Perceived seriousness & .395 & $3.581^{* *}$ & & \\
Perceived changeability & -.064 & -.546 & & \\
Perceived responsibility & -.123 & -1.102 & & \\
Perceived threat & .204 & $1.781^{\wedge}$ & \\
\hline
\end{tabular}

Note. Betas reflect difference scores (depression - migraines), ${ }^{\wedge} p<.10$; $* * p<.005$

through perceivers' beliefs that depression (vs. migraines) is a distressing condition, $B=.20, S E=.06,95 \% \mathrm{CI}[.09, .33]$. The effect size of the indirect effect was $R^{2}=.17, S E=.07$, bootstrap $95 \%$ CI $[.05, .32]$. See Figure $1 .^{3}$

\section{Discussion}

Study 1 showed that perceptions that depression is perceived to be a more distressing condition than migraines contributed to perceivers' greater willingness to recommend treatment for depression (vs. migraines). This effect was not influenced by perceivers' personal (via self or other) familiarity with depression. As predicted, the extent to which perceivers believed that depression was more distressing than migraines mediated the relationship between perceived seriousness and treatment recommendations. Study 2 used neuroimaging to identify the neural processes engaged when nondepressed individuals evaluate depressed individuals. Of interest was whether the neural regions engaged in nondepressed individuals' initial evaluations of depression predicted their behavior (self-reported mental health treatment-seeking intentions).

\section{Study 2}

The two goals of Study 2 were to (1) extend prior neuroimaging work on stigma by demonstrating that nondepressed perceivers engaged a unique pattern of neural activity when they evaluated individuals suffering from depression as

\footnotetext{
${ }^{3}$ Although our mediation analysis suggests that the relationship between beliefs that depression (vs. migraines) is serious and treatment recommendations for depression (vs. migraines) is mediated by distress, it is also plausible that seriousness mediates the relationship between distress and treatment recommendations. We therefore conducted a second mediation analyses using PROCESS with 1,000 bootstrap samples (Hayes, 2012), in which distress was the mediator. Here we found that although distress in response to depression positively predicted treatment recommendations, it acted through the extent of stigma-related beliefs of depression (vs. migraines) as a serious illness, $B=.14, S E=.08,95 \%$ CI $[.03, .34]$.
}

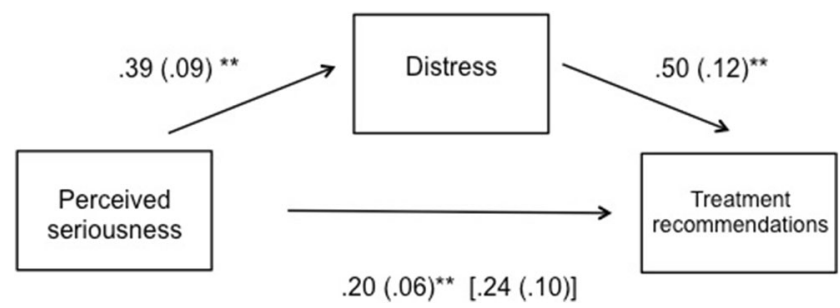

Fig. 1 Results from a mediation analysis demonstrating that participants' beliefs that depression is a more distressing condition than migraines mediated the relationship between their perceptions that depression (versus migraines) is serious and willingness to recommend mental health treatment

compared to a nonstigmatized illness (migraines) or healthy controls (Hypothesis 1); and (2) determine whether perceivers' beliefs that depression is a distressing condition mediated a potential relationship between the neural correlates underlying their initial evaluations and subsequent treatment recommendations.

Stigma is detected rapidly (Krendl et al., 2016), and affects the neural mechanisms engaged by nondepressed perceivers during their initial evaluations of stigmatized individuals (e.g., Harris \& Fiske, 2006; Krendl et al., 2006; Krendl, Kensinger, \& Ambady, 2012; Krendl et al., 2016). We hypothesized that perceivers' initial evaluations of depressed individuals could predict their treatment recommendations in one of two ways. First, perceivers who believe that depression is a distressing condition may engage more dorsomedial prefrontal cortex (dmPFC) activity when they evaluate depressed individuals (suggesting stronger recruitment of impression formation processes; for meta-analyses and reviews, see Denny et al., 2012; Mitchell, 2009; and Van Overwalle, 2009). DmPFC plays a causal role in impression formation (Ferrari et al., 2014; Ferrari, Vecchi, Todorov, \& Cattaneo, 2016), and is engaged regardless of whether impressions are spontaneous or intentional (Ma, Vandekerckhove, Van Overwalle, Seurinck, \& Fias, 2011). Moreover, dmPFC plays a prominent role in forming impressions of dissimilar others (Mitchell, Macrae, \& Banaji, 2006) and evaluating stigmatized individuals (Harris \& Fiske, 2006). If, as predicted, dmPFC is more active when perceivers evaluate depressed (as compared to nondepressed) individuals, then one possibility is that the extent of their dmPFC engagement may predict their willingness to recommend treatment.

It is also possible that perceivers who believe that depression is a distressing condition may engage more regulatory effort when they evaluate depressed individuals. The ventrolateral prefrontal cortex (vlPFC) has been widely implicated in regulating negative emotional responses (Ochsner et al., 2012), as well as in forming impressions of stigmatized individuals (e.g., Krendl, Kensinger, et al., 2012; Krendl et al., 2016). Moreover, the magnitude of vlPFC activation corresponds with perceivers' implicit bias toward stigmatized 
individuals (e.g., Krendl, Heatherton, \& Kensinger, 2009; Krendl, Kensinger, et al., 2012). For this reason, as well as the fact that explicit bias did not predict treatment recommendations in Study 1, we included a measure of implicit bias in Study 2. If, as predicted, vlPFC is more active when perceivers evaluate depressed (as compared to nondepressed) individuals, then another possibility is that the extent of their vlPFC engagement during their initial evaluations would be associated with their bias and would predict their willingness to recommend treatment.

Despite the fact that early treatment seeking predicts the greatest treatment success (e.g., Altamura et al., 2010), an important obstacle to seeking treatment is one's ability to correctly attribute one's symptoms (e.g., feeling down or lethargic) to depression. Moreover, prior research in clinical populations suggests that individuals' attitudes toward mental health treatment play a central role in predicting their actual help-seeking intentions (e.g., Bayer \& Peay, 1997; Mojtabai, Olfson, \& Mechanic, 2002). Thus, to better generalize Study 1, Study 2 assessed self-reported intentions to seek mental health treatment in addition to willingness to recommend mental health treatment for others. We predicted that self-reported treatment-seeking intentions would be positively correlated with mental health treatment recommendations for others, and verified this assumption using the same vignettes as described in Study 1. Further, we predicted that participants' belief that depression is a distressing condition (as measured in the vignettes from Study 1) would influence their own self-reported treatment intentions. These were measured in a separate testing session approximately 1 week prior to the fMRI session.

We predicted that evaluating depressed individuals would elicit heightened activation in dmPFC and/or vlPFC (as compared to evaluating individuals with migraines or healthy controls; Hypothesis 1). Moreover, if nondepressed individuals' neural activity when they evaluated depressed individuals related to their own self-reported treatment-seeking intentions, we anticipated that perceivers' belief that depression (vs. migraines) is a distressing condition would mediate the relationship between either dmPFC (which would suggest greater impression formation) or vlPFC (which would suggest regulation) and self-reported treatment-seeking intentions (Hypothesis 2).

\section{Method}

\section{Participants}

Thirty right-handed White adults with no history of neurological problems (18-29 years of age, 17 female) from Indiana University participated and provided informed consent. This sample size was selected to ensure sufficient power for our analyses (Desmond \& Glover, 2002). Participants were screened to ensure that they had not previously, nor were they currently, suffering from depression. Participation was completed over two testing sessions, approximately 1 week apart. The first session was conducted in the laboratory and consisted of a series of behavioral measures described below. A portion of the behavioral data from one participant was lost due to a computer error. The second session was the fMRI study. Participants completed the fMRI study described below and a separate study unrelated to mental health. Study order was counterbalanced, and no order effects were found. Because stigma interacts with attitudes toward treatment seeking differently as a function of perceivers' race (e.g., Brown et al., 2010; Givens, Katz, Bellamy, \& Holmes, 2007) and ethnicity (e.g., Georg Hsu et al., 2008; Rao, Feinglass, \& Corrigan, 2007), we selected a racially and ethnically homogeneous group for the current study in order to most accurately interpret our results. We chose White participants for two reasons. First, the majority of participants in Study 1 were White $(79 \%)$. Second, the unrelated study was about race, making White participants the preferred participant population for both.

\section{Procedure}

Behavioral testing session Participants completed an fMRI screening and measures relevant to the present task and others in the laboratory approximately 1 week prior to scanning. In this session, we measured participants' treatment recommendations for others and beliefs that depression is a distressing condition (using the same vignettes from Study 1), their selfreported treatment-seeking intentions, and their implicit bias toward depression (described below). Participants also completed the 22-item short version of the Empathy Quotient to control for individual differences in empathy (Wakabayashi et al., 2006).

Assessing participants' self-reported mental health treatment-seeking intentions To measure self-reported intentions toward seeking mental health treatment, participants evaluated three statements describing common symptoms of depression and three statements describing common symptoms of migraines (see Appendix B in the Supplemental Materials for items). Critically, the symptoms for each illness became progressively more serious with each statement (low, moderate, severe). For depression, the first two statements included symptoms from the PHQ-2, a two-item measure assessing depression that, combined, has a sensitivity of $83 \%$ and specificity of $92 \%$ for diagnosing major depression (Kroenke, Spitzer, \& Williams, 2003). The third (highest) symptom severity item specifically addressed suicidality. These items had strong reliability (Cronbach's $\alpha=.84$ ). For migraines, the items were structured to parallel depressive 
symptoms, and were compiled via Internet search engines identifying the most common symptoms of migraines (e.g., experiencing a dull ache on the side of one's head for a few hours per day for multiple days over the past month, having difficulty concentrating on work). The high-severity symptoms also included experiencing symptoms of nausea and vomiting. These items had acceptable reliability (all Cronbach's $\alpha=.72$ ). For each question, participants indicated their likelihood of seeking treatment if they experienced a specific set of symptoms.

We measured participants' willingness to seek treatment for both depression and migraines to control for individual differences in treatment seeking in general. For instance, an individual who is reluctant to seek treatment for any illness would likely have lower treatment intentions for both migraines and depression as compared to an individual who is more willing to seek treatment for any illness. We thus calculated disparity scores between willingness to seek treatment for depression as compared to migraines to use in our subsequent analyses.

In addition to controlling for individual differences in treatment seeking, we were interested in identifying the symptom severity level at which participants were most reluctant to seek treatment for depression (relative to migraines). We did this to increase our sensitivity in detecting whether individual differences in neural activity associated with depression predicted behavior (self-reported treatment intentions for depression specifically). We posited that greater ambivalence for seeking treatment for depression (relative to migraines) would be reflected by higher disparity scores and more variability in the disparity scores for that symptom level.

We thus conducted a 2 (illness: migraines or depression) $\times 3$ (symptom severity: low, moderate, severe) repeatedmeasures ANOVA on the intentions measures. Results revealed a main effect of illness, $F(1,28)=49.63, p<.001$, $\eta_{\mathrm{p}}{ }^{2}=.64$, a main effect of symptom severity, $F(1,28)=$ $126.55, p<.001, \eta_{\mathrm{p}}{ }^{2}=.82$, and an Illness $\times$ Severity interaction, $F(1,28)=11.06, p<.001, \eta_{\mathrm{p}}{ }^{2}=.28$. The main effects emerged because participants had higher intentions to seek treatment for migraines as compared to depression, regardless of symptom severity. They also had higher treatment intentions for both illness types as they increased in symptom severity (see Supplemental Materials for all means). The interaction emerged because the disparity between treatment intentions for migraines and depression differed as a function of symptom severity. Specifically, the disparity was lowest for the high-severity condition $\left(M_{\text {Migraines }}-\right.$ Depression $\left.=.90, S D=1.21\right)$, higher for the low-severity condition $\left(M_{\text {Migraines }}-\right.$ Depression $=1.69, S D=$ $1.44)$, and highest for the moderate-severity condition $\left(M_{\text {Migraines }- \text { Depression }}=2.07, S D=1.60\right)$. This resulted in a significant linear effect, $F(2,56)=11.06, p<.001, \eta_{\mathrm{p}}{ }^{2}=$ .28 . The disparity between the low-severity and moderate- severity conditions did not differ significantly from one another, $t(28)=1.52, p=.14$, but both significantly differed from the high-severity condition (both $t \mathrm{~s}>2.80, p \mathrm{~s}<$ $.01)$. However, of the three symptom severity levels, the moderate-severity condition elicited the most variance in participant responses and also had the highest disparity. Thus, our subsequent analyses used participants' selfreported treatment intentions (depression - migraines) as reported for the moderate-symptom severity level.

Measuring implicit bias Implicit bias was assessed using the Implicit Association Test (IAT; Greenwald, McGhee, \& Schwartz, 1998) that was designed to measure implicit bias toward depression (using the categories depressed/not-depressed, pleasant/unpleasant). Specifically, participants categorized six images of depressed individuals (three male, three female), six images of nondepressed individuals (three male, three female), six pleasant words (e.g., kindness), and six unpleasant words (e.g., jealousy). Participants completed stereotypically congruent (e.g., a pleasant word paired with a nondepressed individual) and incongruent (e.g., a pleasant word paired with a depressed individual) blocks (order was counterbalanced across participants). Consistent with recommended scoring procedures (Greenwald, Nosek, \& Banaji, 2003), we removed trials with less than $200 \mathrm{~ms}$ and over $3,000 \mathrm{~ms}$ and then calculated IAT D (which divides the reaction time difference between incongruent and congruent blocks by the standard deviation of all latencies) to measure implicit bias toward depression.

\section{FMRI stimuli and task}

In the scanner, participants viewed 120 Caucasian faces with neutral expressions drawn from the PAL database (Minear \& Park, 2004). Forty faces were identified as depressed, 40 as migraine afflicted, and 40 as healthy. Faces were equated for attractiveness, distinctiveness, and trustworthiness across conditions (see Cassidy \& Gutchess, 2012). We conveyed diagnosis by placing the face on a red, green, or yellow background (see Cloutier et al., 2011, for a similar approach). The color-diagnosis pairing was counterbalanced across participants, as were faces and diagnosis category. To ensure attention to the color-diagnosis pairings, participants reported at three separate time points (after instructions, the first scan, and immediately posttask) which diagnosis was paired with which color. All participants successfully did this at every assessment point.

The task was modeled as an event-related design over one run that lasted 5 minutes and 38 seconds $(169 \mathrm{TRs} ; 1 \mathrm{TR}=2 \mathrm{~s})$. Participants viewed each face for 2 seconds and indicated via button press how much they liked the individual pictured on a 4-point scale ( 1 = highly dislike, 4 = highly like). We asked 
participants to indicate how much they would like the targets because prior work has shown that dmPFC is engaged during impression formation regardless of task instructions, but vlPFC is engaged during intentional impression formation (Ma et al., 2011). Our design optimized the likelihood of detecting activity in both regions. Images were randomly presented, with no more than two of the same diagnosis type sequentially. Periods of jitter, in the form of a fixation cross at the center of the display, ranged from 2 to 8 seconds. Participant responses were monitored to ensure attention during the task.

\section{Data acquisition and analysis}

Whole-brain imaging was performed on a Siemens 3.0T TIM Trio MRI scanner at the Indiana University Imaging Research Facility in Bloomington, Indiana. Anatomical images were acquired with a high-resolution 3-D magnetization-prepared rapid gradient-echo sequence (224 slices; echo time $[\mathrm{TE}]=3.02 \mathrm{~ms}$; repetition time $[\mathrm{TR}]=2,200 \mathrm{~ms}$; flip angle $=9^{\circ} ; .8 \times .8 \times .8$-mm voxels $)$. Functional images were collected over one run of 169 time points, using a fast field echo-planar sequence sensitive to blood-oxygen-level-dependent contrast (T2*; 32 slices with 3.5-mm thickness and 3.5-mm skip; TR = 2,000 ms; $\mathrm{TE}=30 \mathrm{~ms}$; flip angle $=70^{\circ}$ ).

Preprocessing and analyses of functional data were conducted in SPM8 (Wellcome Trust Centre for Neuroimaging, London, UK). Images were realigned to correct for motion, normalized to the MNI (Montreal Neurological Institute) template, and smoothed using a 6-mm FWHM isotropic Gaussian kernel. We used custom artifact detection software to detect motion artifact after preprocessing (http://www.nitrc.org/projects/artifact detect) in individual runs on a participant-by-participant basis. We applied a priori motion criteria that participants be excluded if they moved more than $2 \mathrm{~mm}$ during the functional run or that individual volumes be removed (as nuisance regressors) if head motion on that volume exceeded $1 \mathrm{~mm}$ or the overall signal for that time point fell three standard deviations outside the mean global signal for the entire run. None of the participants met either exclusionary criteria, and therefore no participant or volume was removed.

Data were resampled to $3-\mathrm{mm}$ isotropic voxels in a $96 \times$ 96 matrix. A general linear model incorporating the three image types (faces of ostensibly depressed, migraineafflicted, or healthy individuals) and covariates of no interest (a session mean, a linear trend, and six movement parameters derived from realignment corrections) computed parameter estimates $(\beta)$ and $t$-contrast images (containing weighted parameter estimates) for each comparison at each voxel and for each participant. We conducted a whole-brain ANOVA, with condition (condition: depressed, migraines, healthy) as the independent variable.

Individual differences were examined using region of interest (ROI) analyses based on the results from the wholebrain ANOVA. For all ROI analyses, we averaged the parameter estimates from the $8-\mathrm{mm}$ sphere surrounding the peak coordinate of interest (see fMRI results for specific peaks) using the relevant condition-baseline contrast. For the ANOVA, we used an extent threshold of $p<.001$, with a 10-voxel extent threshold correction (see Lieberman \& Cunningham, 2009).

\section{Results}

\section{Behavioral results}

Recommending treatment and distress for depressed others predict self-reported treatment seeking intentions As in Study 1, perceivers did not differ in how distressing they found having migraines or depression to be based on the vignettes $\left(M_{\text {Depression }}=5.72, S D=1.07 ; M_{\text {Migraines }}=\right.$ 5.69, $S D=1.04, t<1$ ); how much sympathy they had for individuals with either illness $\left(M_{\text {Depression }}=5.83, S D=\right.$ $\left.1.39 ; M_{\text {Migraines }}=5.83, S D=1.20, t<1\right)$; or their willingness to recommend treatment for either illness $\left(M_{\text {Depression }}=6.17, S D=.89 ; M_{\text {Migraines }}=6.45, S D=\right.$ $.78), t(28)=1.77, p=.09$. Also as in Study 1, perceivers' ratings for migraines and depression were highly correlated with each other-sympathy: $r(29)=.56, p<.005$; distress $r(29)=.63, p<.001$; treatment recommendation: $r(29)=.50, p<.01$.

We used difference scores (depression - migraines) for all reported analyses (see Study 1 for rationale). An important goal of Study 2 was to better generalize treatment recommendations for others to participants' own selfreported treatment intentions because clinical research suggests that attitudes toward mental health treatment play a central role in predicting actual help-seeking intentions (e.g., Bayer and Peay, 1997; Mojtabai et al., 2002). To verify this, we examined whether self-reported treatment seeking intentions (for depression - migraines) and treatment recommendations for others (depression vignettes migraines vignettes) were related. Self-reported treatment seeking intentions and treatment recommendations for others from the vignettes were positively correlated, $r(29)=.41, p=.027$. Moreover, perceptions that depression (vs. migraines) is a distressing condition predicted self-reported treatment-seeking intentions for depression (vs. migraines), $r(29)=.57, p=.001^{4}$

\footnotetext{
${ }^{4}$ It is worth noting that self-reported treatment intentions for the low symptom severity did not correlate with treatment recommendations, $r(28)=.22, p=$ .25 .
} 
Likability ratings during the fMRI task We conducted a repeated-measures ANOVA to determine whether participants' explicit likeability ratings during the scanning session differed by diagnosis (depressed, migraines, healthy). A marginal effect emerged, $F(1,29)=3.112, p=.088, \eta_{\mathrm{p}}{ }^{2}$ $=.097$. Participants had slightly lower liking ratings for depressed and migraine-afflicted individuals $\left(M_{\text {Depressed }}=\right.$ $2.419, S D=.287 ; M_{\text {Migraine }}=2.440, S D=.310$ ) versus healthy individuals $\left(M_{\text {Healthy }}=2.545, S D=.370\right)$. Because these results did not reach traditional levels of significance, they should be cautiously interpreted.

\section{fMRI results}

\section{Hypothesis 1: DmPFC and vlPFC are more active when} nondepressed participants form impressions of depressed individuals A whole-brain ANOVA identified neural activity differing by diagnosis (healthy, depression, migraines). The ANOVA revealed main effects in the dmPFC (BA 9: 0, 33,39 ), right VLPFC (BA 47: 51, 36, -6), and left superior frontal gyrus (BA 9: -21, 60, 30). As expected, subsequent ROI analyses revealed that activation in these regions was driven by the depression diagnosis condition. Specifically, evaluating depressed (vs. migraine-afflicted or healthy) individuals elicited heightened activation in these regions (see Table 2 and Fig. 2). For t-tests directly comparing each condition, see Table 3.

\section{Hypothesis 2: Distress toward depression mediates the re-} lationship between dmPFC, but not vIPFC, activity and self-reported treatment intentions We next tested whether distress mediated a relationship between dmPFC or vlPFC activity and perceivers' self-reported mental health treatment-seeking intentions. Here, we used the dmPFC and vlPFC peaks that emerged from the main effect of diagnosis condition in the whole-brain ANOVA (dmPFC: $0,33,39$ ), and (vlPFC: $51,36,-6$ ). Since dmPFC or vlPFC activity could also mediate a relationship between distress and self-reported mental health treatment-seeking intentions, we tested both models using PROCESS in SPSS with 1,000 bootstrap samples (Hayes, 2012). As in Study 1 , we used difference scores (depression - migraines) for all analyses. Thus, the difference scores identified patterns of neural activation that may have differed when perceivers evaluated depressed individuals as compared to when they evaluated individuals suffering from migraines.

In our first model, significant indirect effects emerged between dmPFC activation and self-reported intentions to seek treatment. As predicted, although dmPFC response when evaluating depressed individuals positively predicted self-reported treatment intentions (for depression vs. migraines), distress mediated this relationship, $B=.22, S E$ $=.16,95 \% \mathrm{CI}[.04, .67]$. The effect size of the indirect effect was $R^{2}=.12, S E=.06$, bootstrap $95 \%$ CI $[.02$, .26] (see Fig. 3). ${ }^{5}$

The extent of vlPFC activity in response to evaluating individuals suffering from depression as compared to migraines was positively correlated with dmPFC activity: $r(30)=.61, p$ $<.001$, and marginally correlated with perceivers' implicit bias (IAT D): $r(30)=-.35, p=.060$. However, it was not related to their self-reported treatment intentions: $r(30)=$ $.01, p=.98$. Similarly, the model examining whether perceived distress mediated a potential relationship between vlPFC activation and self-reported treatment intentions was not significant, $B=.37, S E=.35,95 \%$ CI $[-.14,1.23]$. The effect size of the indirect effect was $R^{2}=-.02, S E=.05$, bootstrap 95\% CI [-.19, .03].

\section{Discussion}

Study 2 revealed two key findings. First, perceivers had increased dmPFC and vlPFC activation when evaluating depressed (vs. migraine-afflicted and healthy) individuals (Hypothesis 1). Second, perceivers who had a greater extent of engagement in impression formation processes (dmPFC) when they evaluated individuals suffering from depression (as compared to migraines) had a smaller difference between their self-reported treatment-seeking intentions for depression as compared to migraines. Perceptions that depression is a more distressing condition than migraines mediated the relationship for $\mathrm{dmPFC}$ and treatment recommendations (Hypothesis 2). Activity in the vlPFC was not associated with self-reported treatment-seeking intentions, but it was marginally associated with perceivers' implicit bias (with more bias predicting less vlPFC engagement for depression vs. migraines).

\section{General discussion}

The present work identified the first potential neurobiological mechanism for how neural activity associated with evaluating depressed individuals predicts nondepressed perceivers' selfreported mental health treatment-seeking intentions. Specifically, dmPFC activity in response to evaluating

\footnotetext{
$\overline{5}$ We also conducted the mediation analysis using participants' self-reported intention to seek treatment for the low-symptom severity level. Here, although $\mathrm{dmPFC}$ activation positively predicted self-reported treatment intentions, $r(28)$ $=.43, p=.021$, distress was not significantly related to treatment intentions for low-symptom severity, $r(28)=.27, p=.17$. Finally, we examined whether perceivers' stigma-related beliefs that depression is a serious illness (which was the only belief that predicted their willingness to recommend treatment in Study 1) mediated the relationship between dmPFC activation and selfreported treatment seeking intentions at the moderate symptom severity. The indirect effect in this model was not significant: $B=.003, S E=.05,95 \% \mathrm{CI}$ $[-.06, .17]$. The effect size of the indirect effect was $R^{2}=.002, S E=.03$, bootstrap 95\% CI [-.04, .08].
} 
Table 2 Main effects from whole-brain voxel-wise ANOVA

\begin{tabular}{|c|c|c|c|c|c|}
\hline Brain region & $x$ & $y$ & $z$ & Kextent & $t$ score \\
\hline \multicolumn{6}{|l|}{ Depressed $>$ Healthy + Migraines } \\
\hline Left superior frontal gyrus (BA 9) & -21 & 60 & 30 & 18 & 4.27 \\
\hline Right vlPFC (BA 47) & 51 & 36 & -9 & 17 & 4.11 \\
\hline Right dmPFC (BA 9) & 3 & 36 & 33 & 35 & 4.01 \\
\hline Left middle frontal gyrus (BA 47) & -48 & 36 & -3 & 21 & 3.7 \\
\hline Left vlPFC (BA 47) & -42 & 18 & -12 & 10 & 3.97 \\
\hline Left cuneus (BA 18) & -3 & -84 & 18 & 612 & 5.72 \\
\hline Right cuneus (BA 17) & 9 & -81 & 9 & $*$ & 5.21 \\
\hline Right posterior cingulate (BA 30) & 15 & -63 & 12 & $*$ & 5.19 \\
\hline \multicolumn{6}{|l|}{ Healthy $>$ Depressed + Migraines } \\
\hline Right insula (BA 13) & 33 & -3 & 15 & 14 & 5.6 \\
\hline Left parahippocampal gyrus (BA 36) & -33 & -27 & -21 & 11 & 4.91 \\
\hline Left inferior occipital gyrus (BA 18) & -36 & -90 & -6 & 15 & 4.04 \\
\hline Left lingual gyrus (BA 17) & -12 & -96 & -9 & 14 & 3.91 \\
\hline \multicolumn{6}{|l|}{ Migraines $>$ Depressed + Healthy } \\
\hline No significant voxels & & & & & \\
\hline
\end{tabular}

Organized from anterior to posterior. * indicates subclusters. $p<.001$ uncorrected, 10 voxel extent threshold, all coordinates Montreal Neurological Institute

depressed individuals predicted self-reported treatment-seeking intentions for depression. However, this relationship acted through distress. These findings have direct relevance to advancing our understanding of how stigma-related beliefs about depression impact nondepressed perceivers' willingness to recommend or seek mental health treatment.

Our results suggest that stronger engagement of dmPFC positively predicts perceivers' self-reported treatment-seeking intentions. In order to determine whether the dmPFC peak observed in the current study was a region implicated in evaluating others, we examined a meta-analysis on studies examining the neural correlates underlying judgments about others (vs. self; Denny et al., 2012). We found that the dmPFC activity we observed in the current study is indeed similar to the dmPFC region implicated by the meta-analysis in evaluating others. One potential explanation for this finding is that when

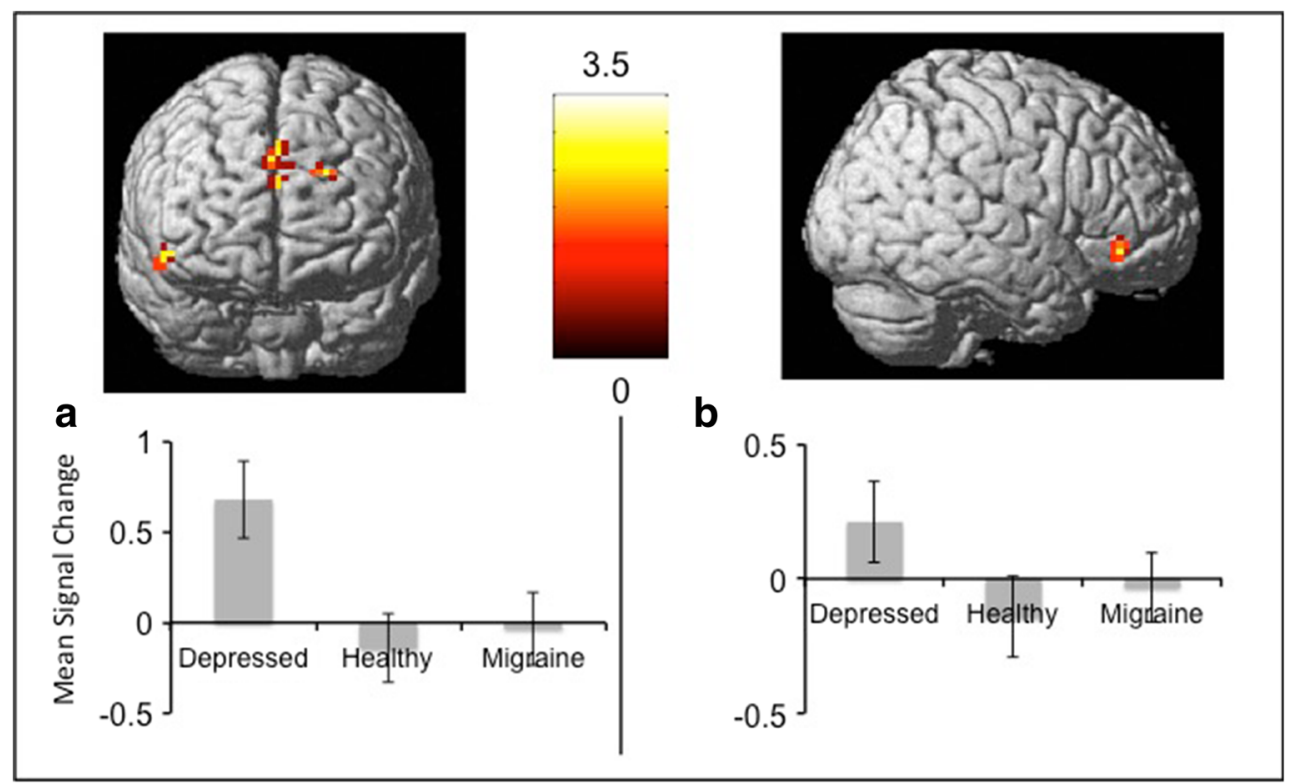

Fig. 2 Anterior and lateral views of the main effects emerging from the whole brain voxel-wise three-way ANOVA (Depression, Migraines, Healthy), $p$ $<.05$ corrected. Subsequent region-of-interest analyses plotted for the dmPFC (a) and vlPFC (b). Error bars are SEM 
Table $3 T$ tests comparing (a) depressed versus migraines; (b) depressed versus healthy; and (c) migraines versus healthy

\begin{tabular}{|c|c|c|c|c|c|}
\hline Brain region & $x$ & $y$ & $z$ & Kextent & $t$ score \\
\hline \multicolumn{6}{|l|}{ a Depressed > Migraines } \\
\hline Left superior frontal gyrus (BA 9) & -21 & 60 & 30 & 26 & 4.33 \\
\hline Right dmPFC (BA 8) & 12 & 39 & 45 & 26 & 4.88 \\
\hline Right vlPFC (BA 47) & 51 & 36 & -15 & 44 & 5.49 \\
\hline Left dmPFC (BA 8) & -15 & 36 & 54 & 10 & 3.93 \\
\hline Left vIPFC (BA 11/47) & -42 & 33 & -3 & 23 & 4.06 \\
\hline Left superior frontal gyrus (BA 6) & -12 & 15 & 60 & 20 & 4.97 \\
\hline Left middle frontal gyrus (BA 6) & -42 & 9 & 51 & 28 & 4.52 \\
\hline Left caudate & -18 & -3 & 18 & 38 & 5.11 \\
\hline Right middle temporal gyrus (BA 22) & 57 & -45 & 3 & 10 & 4.29 \\
\hline Left precuneus (BA 7) & -6 & -60 & 30 & 70 & 5.89 \\
\hline Left angular gyrus (BA 39) & -48 & -60 & 33 & 53 & 4.83 \\
\hline Right posterior cingulate (BA 30) & 18 & -66 & 9 & 380 & 5.39 \\
\hline Right cerebellum & 30 & -66 & -18 & 20 & 4.26 \\
\hline Right cerebellum & 60 & -81 & -15 & 10 & 4.77 \\
\hline \multicolumn{6}{|l|}{ Migraines $>$ Depressed } \\
\hline \multicolumn{6}{|l|}{ No significant voxels } \\
\hline \multicolumn{6}{|l|}{$\boldsymbol{b}$ Depressed $>$ Healthy } \\
\hline Left superior frontal gyrus (BA 9) & -21 & 60 & 30 & 26 & 4.33 \\
\hline Right dmPFC (BA 8) & 12 & 39 & 45 & 26 & 4.88 \\
\hline Right vlPFC (BA 47) & 51 & 36 & -15 & 44 & 5.49 \\
\hline Left dmPFC (BA 8) & -15 & 36 & 54 & 10 & 3.93 \\
\hline Left vlPFC (BA 11/47) & -42 & 33 & -3 & 23 & 4.06 \\
\hline \multicolumn{6}{|l|}{ Healthy $>$ Depressed } \\
\hline \multicolumn{6}{|l|}{ No significant voxels } \\
\hline \multicolumn{6}{|l|}{ c Migraines $>$ Healthy } \\
\hline \multicolumn{6}{|l|}{ No significant voxels } \\
\hline \multicolumn{6}{|l|}{ Healthy $>$ Migraines } \\
\hline Left putamen & -21 & -9 & 12 & 29 & 5.29 \\
\hline Left cuneus (BA 23) & -9 & -72 & 12 & 17 & 4.67 \\
\hline
\end{tabular}

$p<.001$ uncorrected, 10 voxel extent threshold, all coordinates Montreal Neurological Institute perceivers evaluate depressed individuals, the extent to which they engage neural processes associated with evaluating others may be associated either with reduced dehumanization

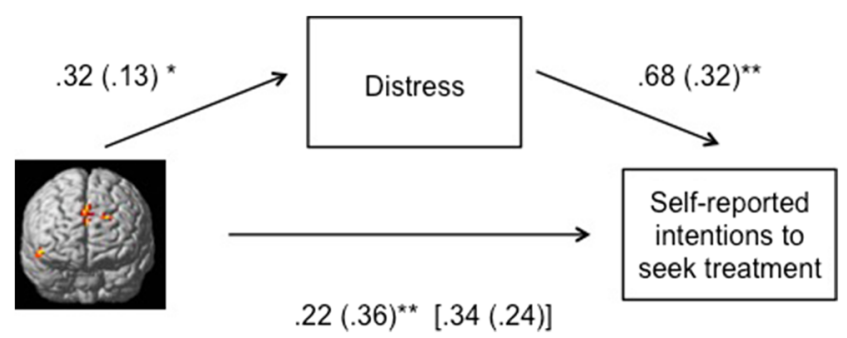

Fig. 3 Results from a mediation analysis demonstrating that participants' beliefs that depression is a more distressing condition than migraines mediated the relationship between neural activity in the dorsomedial prefrontal cortex (in response to viewing a depressed individual as compared to an individual suffering from migraines) and self-reported treatment seeking intentions or greater individuation of depressed individuals. For instance, Harris and Fiske (2006) found that nonstigmatized perceivers had reduced activation in the dmPFC when they evaluated highly stigmatized individuals (e.g., homeless individuals), and suggested that this may provide insight into why highly stigmatized groups are dehumanized (e.g., Fiske et al., 2002). Being humanized has been associated with many interpersonal benefits, such as a heightened moral status (Bastian, Laham, Wilson, Haslam, \& Koval, 2011), fairer treatment, and greater empathy (Cehajic, Brown, \& Gonzalez, 2009). Dehumanization of outgroup members reverses these effects and is associated with greater aggression (Viki, Osgood, \& Phillips, 2013), discrimination (Pereira, Vala, \& Leyens, 2009), and harsher punishments (Fincher \& Tetlock, 2016) toward dehumanized individuals. DMPFC engagement may therefore be an important link between evaluation and behavior because it may reflect perceivers' increased humanization 
of depressed individuals and thus greater willingness to recommend treatment for those individuals.

In addition to increased activity in dmPFC, participants also had heightened vlPFC activation when they evaluated depressed individuals. Although vlPFC activity did not predict distress or treatment-seeking intentions for depression versus migraines, it was positively associated with $\mathrm{dmPFC}$ activity and had a marginally negative association with implicit bias. One speculative possibility is that this region, which has been widely implicated regulating negative responses (for review, see Amodio, 2014; see also Cassidy, Lee, \& Krendl, 2016; Krendl et al., 2012), may play a role in downregulating perceivers' stigma-related bias during initial evaluations. Given that treatment recommendations were not directly predicted by explicit bias in measured Study 1 , and stigmatized individuals elicit multiple parallel neural responses during impression formation (e.g., Harris \& Fiske, 2006; Krendl et al., 2012; Krendl et al., 2006)—some of which are associated with bias (e.g., Krendl, Kensinger, et al., 2012)-future research should further investigate this question.

Study 1 demonstrated that perceivers' beliefs that depression is a serious illness corresponded with increased beliefs that depression is a distressing condition. Moreover, perceived seriousness mediated the relationship between distress and treatment recommendations. However, Study 2 found that only perceptions that depression is more distressing than migraines (not that it is more serious; see footnote 5) predicted greater $\mathrm{dmPFC}$ activity when evaluating depressed versus migraine-afflicted individuals. One possible explanation for this finding is that recommending treatment for depressed (vs. migraine-afflicted) individuals (irrespective of perceivers' own experience with depression) may depend on perceivers' beliefs about the affective impact associated with suffering from depression (distress), rather than their specific stigmarelated beliefs (seriousness). Future research should investigate this possibility.

There are several limitations to the current study that reduce the overall generalizability of our findings. First, using difference scores in Studies 1 and 2 limited the interpretability of our results. Although we intentionally used difference scores to control for individual differences that would affect treatment recommendations for any disorder (e.g., an inclination to perceive all illnesses as being serious), an important consequence of this decision is that our findings can be interpreted only in the context of migraines. For instance, it would not be accurate to posit that Study 1 suggests that perceptions that depression is a serious illness predict treatment recommendations. Rather, our findings show that perceptions that depression is a more serious illness than migraines predict greater willingness to recommend treatment for depression than for migraines. It is worth noting that results from Study 1 hold when we used the depression ratings without the difference scores. ${ }^{6}$ Our rationale for using difference scores in the current study was based on the assumption that people tend to view depression as being different from disorders with more "physical" symptoms. This assumption was verified by the findings in the current studies.

Relatedly, instead of using difference scores for our analyses, we could have also controlled for perceivers' migraine ratings in our regressions. However, because we had based our power analyses for Study 1 on the six predictor variables we tested in the regression (bias, familiarity, and four stigma beliefs about depression), we were underpowered to conduct a regression with five additional predictors (e.g., beliefs and familiarity toward migraines). That said, the same pattern of results emerged when the Study 1 regression was run, predicting treatment recommendations for depression without using difference scores but instead including migraines as predictors in the model.

A third limitation in the current investigation is that Study 2 only examined White participants. This was because prior work suggests that stigma interacts with attitudes toward treatment seeking differently as a function of perceivers' race (e.g., Brown et al., 2010; Givens et al., 2007) and ethnicity (e.g., Georg Hsu et al., 2008; Rao et al., 2007). Although Study 1 included some non-White participants, the findings are unchanged when analyzed with only White participants (see footnote 2 ). Thus, although our results may speak to effects relevant to White college students (our target population), we cannot speak to whether or not they generalize to other populations. Future research should extend these findings to other racial and ethnic groups as well as to other age groups.

The current studies enhance our knowledge of the manner in which initial evaluations influence important outcomes such as nondepressed individuals' treatment recommendations. Although future work should extend these findings to clinical populations, our results provide the initial characterization of how stigma affects treatment-seeking intentions. Even though the individuals in the current study had never experienced depression, assessing how stigma affects their treatment-seeking intentions directly informs efforts to reduce effects of stigma on endorsing treatment. The present research identified a link between neural activity reflective of greater engagement of impression formation processes when evaluating depressed individuals and subsequent mental health treatment recommendations. Importantly, because increased

\footnotetext{
${ }^{6}$ Predicting treatment recommendations for depression (without using the difference scores) from the four beliefs (seriousness, responsibility, threat, and changeability) when controlling for familiarity and bias is significant, $F(6,68)=5.31, p<.001$, and accounted for $33.2 \%$ of the overall variance. When predicting seriousness from sympathy and distress using the depression ratings in Study 1 (without using difference scores), the model is significant, $F(2,69)=30.35, p<.001$, and accounted for $47.5 \%$ of the overall variance (compared to $24 \%$ when using the difference scores).
} 
engagement of impression formation has been implicated in increased humanization of stigmatized targets (e.g., Harris \& Fiske, 2006), these findings may inform the development of future interventions that promote more positive beliefs about mental health treatment (e.g., emphasizing depression's seriousness). Such an intervention has the ability to significantly improve the quality of life and well-being of individuals suffering from depression.

Acknowledgements The authors thank Bryan Bennett, Gregory Sprout, and Meredith Boyd for research assistance. This project was supported by the American Psychological Foundation Visionary Grant and a grant from the Vice Provost for Research at Indiana University through the Faculty Research Support Program to A.C.K. and by NIA Grant F32AG051304 to B.S.C.

\section{References}

Altamura, A. C., Dell'Osso, B., Berlin, H., Buoli, M., Bassetti, R., \& Mundo, E. (2010). Duration of untreated illness and suicide in bipolar disorder: A naturalistic study. European Archives of Psychiatry and Clinical Neuroscience, 260, 385-391. https://doi. org/10.1007/s00406-009-0085-2

Ambady, N., \& Rosenthal, R. (1992). Thin slices of expressive behavior as predictors of interpersonal consequences: A meta-analysis. Psychological Bulletin, 111, 256-274. http://dx.doi.org/10.1037/ 0033-2909.111.2.256

Amodio, D. M. (2014). The neuroscience of prejudice and stereotyping. Nature Reviews Neuroscience. https://doi.org/10.1038/nrn3800

Bargh, J. A., \& Pietromonaco, P. (1982). Automatic information processing and social perception: The influence of trait information presented outside of conscious awareness on impression formation. Journal of personality and Social psychology, 43(3), 437.

Bastian, B., Laham, S. M., Wilson, S., Haslam, N., \& Koval, P. (2011). Blaming, praising, and protecting our humanity: The implications of everyday dehumanization for judgments of moral status. British Journal of Social Psychology, 50(3), 469-483.

Batson, C. D., Early, S., \& Salvarani, G. (1997). Perspective taking: Imagining how another feels versus imaging how you would feel. Personality and Social Psychology Bulletin, 23(7), 751-758.

Batson, C. D., Fultz, J., \& Schoenrade, P. A. (1987). Distress and empathy: Two qualitatively distinct vicarious emotions with different motivational consequences. Journal of Personality, 55(1), 19-39.

Bayer, J. K., \& Peay, M. Y. (1997). Predicting intentions to seek help from professional mental health services. Australian and New Zealand Journal of Psychiatry, 31(4), 504-513.

Brewer, M. B. (1988). A dual process model of impression formation. In T. Srull, \& R. S. Wyer (Eds.), Advances in social cognition (Vol. 1, pp. 1-36). Hillsdale: Erlbaum.

Brown, C., Conner, K. O., Copeland, V. C., Grote, N., Beach, S., Battista, D., \& Reynolds, C. F. (2010). Depression stigma, race, and treatment seeking behavior and attitudes. Journal of Community Psychology, 38(3), 350-368.

Cassidy, B. S., \& Gutchess, A. H. (2012). Social relevance enhances memory for impressions in older adults. Memory, 20(4), 332-345.

Cassidy, B. S., \& Krendl, A. C. (2016). Dynamic neural mechanisms underlie race disparities in social cognition. NeuroImage, 132, 238-246.

Cehajic, S., Brown, R., \& Gonzalez, R. (2009). What do I care? Perceive ingroup responsibility and dehumanization as predictors of empathy felt for the victim group. Group Processes and Intergroup Relations, $12,715-729$.

Centers for Disease Control and Prevention (2013). Ten Leading Causes of Death by Age Group, United States -2013. Retrieved from: http:// www.cdc.gov/injury/wisqars/pdf/leading_causes_of_death_by_ age_group_2013-a.pdf

Cloutier, J., Gabrieli, J. D., O'Young, D., \& Ambady, N. (2011). An fMRI study of violations of social expectations: When people are not who we expect them to be. NeuroImage, 57(2), 583-588.

Corrigan, P. (2004). How stigma interferes with mental health care. American Psychologist, 59(7), 614.

Crisp, A. H., Gelder, M. G., Rix, S., Meltzer, H. I., \& Rowlands, O. J. (2000). Stigmatisation of people with mental illnesses. The British Journal of Psychiatry, 177(1), 4-7.

Deaux, K., Reid, A., Mizrahi, K., \& Ethier, K. A. (1995). Parameters of social identity. Journal of Personality and Social Psychology, 68(2), 280.

Denny, B. T., Kober, H., Wager, T. D., \& Ochsner, K. N. (2012). A metaanalysis of functional neuroimaging studies of self-and other judgments reveals a spatial gradient for mentalizing in medial prefrontal cortex. Journal of Cognitive Neuroscience, 24(8), 1742-1752.

Desmond, J. E., \& Glover, G. H. (2002). Estimating sample size in functional MRI (fMRI) neuroimaging studies: Statistical power analyses. Journal of Neuroscience Methods, 118(2), 115-128.

Devine, P. G. (1989). Stereotypes and prejudice: Their automatic and controlled components. Journal of Personality and Social Psychology, 56(1), 5.

Faul, F., Erdfelder, E., Lang, A., \& Buchner, A. (2007). G*Power 3: A flexible statistical power analysis program for the social, behavioral, and biomedical sciences. Behavior Research Methods, 39(2), 175191.

Feldman, D. B., \& Crandall, C. S. (2007). Dimensions of mental illness stigma: What about mental illness causes social rejection?. Journal of Social and Clinical Psychology, 26(2), 137-154.

Ferrari, C., Lega, C., Vernice, M., Tamietto, M., Mende-Siedlecki, P., Vecchi, T., ... Cattaneo, Z. (2014). The dorsomedial prefrontal cortex plays a causal role in integrating social impressions from faces and verbal descriptions. Cerebral Cortex. https://doi.org/10.1093/ cercor/bhu186

Ferrari, C., Vecchi, T., Todorov, A., \& Cattaneo, Z. (2016). Interfering with activity in the dorsomedial prefrontal cortex via TMS affects social impressions updating. Cognitive, Affective, \& Behavioral Neuroscience, 16(4), 626-634.

Fincher, K., \& Tetlock, P. (2016). Dehumanization of faces is activated by norm violations and facilitates norm enforcement. Journal of Experimental Psychology: General, 145(2), 131-146.

Fiske, S. T., Cuddy, A. J., Glick, P., \& Xu, J. (2002). A model of (often mixed) stereotype content: Competence and warmth respectively follow from perceived status and competition. Journal of Personality and Social Psychology, 82(6), 878.

Fiske, S. T., \& Neuberg, S. L. (1990). A continuum of impression formation, from category- based to individuating processes: Influences of information and motivation on attention and interpretation. Advances in Experimental Social Psychology, 23, 1-74.

Georg Hsu, L. K., Wan, Y. M., Chang, H., Summergrad, P., Tsang, B. Y., \& Chen, H. (2008). Stigma of depression is more severe in Chinese Americans than Caucasian Americans. Psychiatry: Interpersonal and Biological Processes, 71(3), 210-218.

Givens, J. L., Katz, I. R., Bellamy, S., \& Holmes, W. C. (2007). Stigma and the acceptability of depression treatments among African Americans and whites. Journal of General Internal Medicine, 22(9), 1292-1297.

Goffman, E. (1963). Stigma: Notes on the management of spoiled identity. New York: Simon and Schuster. 
Greenwald, A. G., McGhee, D. E., \& Schwartz, J. L. (1998). Measuring individual differences in implicit cognition: The implicit association test. Journal of Personality and Social Psychology, 74(6), 1464.

Greenwald, A. G., Nosek, B. A., \& Banaji, M. R. (2003). Understanding and using the implicit association test: I. An improved scoring algorithm. Journal of Personality and Social Psychology, 85(2), 197.

Harris, L. T., \& Fiske, S. T. (2006). Dehumanizing the lowest of the low neuroimaging responses to extreme out-groups. Psychological Science, 17(10), 847-853.

Hayes, A. F. (2012). PROCESS: A versatile computational tool for observed variable mediation, moderation, and conditional process modeling [White paper]. Retrieved from Retrieved from http:// www.afhayes.com/public/process2012.pdf

Hinshaw, S. P. (2006). The mark of shame: Stigma of mental illness and an agenda for change. Oxford: Oxford University Press.

Janz, N. K., \& Becker, M. H. (1984). The health belief model: A decade later. Health Education \& Behavior, 11(1), 1-47.

Krendl, A. C., Heatherton, T. F., \& Kensinger, E. A. (2009). Aging minds and twisting attitudes: An fMRI investigation of age differences in inhibiting prejudice. Psychology and Aging, 24(3), 530.

Krendl, A.C., Kensinger, E.A., \& Ambady, N. (2012). How does the brain regulate negative bias to stigma? Social Cognitive \& Affective Neuroscience. Advance online publication. https://doi. org $/ 10.1093 /$ scan $/$ nsr046

Krendl, A. C., Macrae, C. N., Kelley, W. M., Fugelsang, J. A., \& Heatherton, T. F. (2006). The good, the bad, and the ugly: An fMRI investigation of the functional anatomic correlates of stigma. Social Neuroscience, 1(1), 5-15.

Krendl, A. C., Moran, J. M., \& Ambady, N. (2012). Does context matter in evaluations of stigmatized individuals? An fMRI study. Social Cognitive and Affective Neuroscience. https://doi.org/10.1093/ scan/nss037

Krendl, A. C., Zucker, H. R., \& Kensinger, E. A. (2016). Identifying social stigma in $340 \mathrm{~ms}$ : Examining the effects of emotion regulation on the ERP response to negative social stimuli. Social Neuroscience, 12(3), 1-12.

Kroenke, K., Spitzer, R. L., \& Williams, J. B. (2003). The Patient Health Questionnaire-2: validity of a two-item depression screener. Medical Care, 41(11), 1284-1292.

Leventhal, H., Diefenbach, M., \& Leventhal, E. A. (1992). Illness cognition: Using common sense to understand treatment adherence and affect cognition interactions. Cognitive Therapy and Research, 16(2), 143-163.

Lieberman, M. D., \& Cunningham, W. A. (2009). Type I and Type II error concerns in fMRI research: Re-balancing the scale. Social Cognitive and Affective Neuroscience, 4(4), 423.

Link, B. G., \& Phelan, J. C. (2006). Stigma and its public health implications. The Lancet, 367(9509), 528-529.

Ma, N., Vandekerckhove, M., Van Overwalle, F., Seurinck, R., \& Fias, W. (2011). Spontaneous and intentional trait inferences recruit a common mentalizing network to a different degree: Spontaneous inferences activate only its core areas. Social Neuroscience, 6(2), 123-138.

Major, B., \& O'Brien, L. T. (2005). The social psychology of stigma. Annual Review of Psychology, 56, 393-421.

Mende-Siedlecki, P., Cai, Y., \& Todorov, A. (2012). The neural dynamics of updating person impressions. Social Cognitive and Affective Neuroscience. https://doi.org/10.1093/scan/nss040
Minear, M., \& Park, D. C. (2004). A lifespan database of adult facial stimuli. Behavior Research Methods, Instruments, \& Computers, $36(4), 630-633$.

Mitchell, J. P. (2009). Social psychology as a natural kind. Trends in Cognitive Sciences, 13(6), 246-251.

Mitchell, J. P., Macrae, C. N., \& Banaji, M. R. (2006). Dissociable medial prefrontal contributions to judgments of similar and dissimilar others. Neuron, 50(4), 655-663.

Mojtabai, R., Olfson, M., \& Mechanic, D. (2002). Perceived need and help-seeking in adults with mood, anxiety, or substance use disorders. Archives of General Psychiatry, 59, 77-84.

Moses, T. (2010). Being treated differently: Stigma experiences with family, peers, and school staff among adolescents with mental health disorders. Social Science \& Medicine, 70(7), 985-993.

National Institute of Mental Health. (2012). Substance abuse and mental health services administration. Results from the 2009 National Survey on Drug Use and Health: Mental health findings. Rockville: Center for Behavioral Health Statistics and Quality.

Ochsner, K. N., Silvers, J. A., \& Buhle, J. T. (2012). Functional imaging studies of emotion regulation: a synthetic review and evolving model of the cognitive control of emotion. Annals of the New York Academy of Sciences (New York, NY), 1251(1), E1-E24.

Pereira, C., Vala, J., \& Leyens, J. P. (2009). From infra-humanization to discrimination: The mediation of symbolic threat needs egalitarian norms. Journal of Experimental Social Psychology, 45(2), 336-344.

Pescosolido, B. A., Martin, J. K., Lang, A., \& Olafsdottir, S. (2008). Rethinking theoretical approaches to stigma: A framework integrating normative influences on stigma (FINIS). Social Science \& Medicine, 67(3), 431-440.

Rao, D., Feinglass, J., \& Corrigan, P. (2007). Racial and ethnic disparities in mental illness stigma. The Journal of nervous and mental disease, 195(12), 1020-1023.

Richeson, J. A., Baird, A. A., Gordon, H. L., Heatherton, T. F., Wyland, C. L., Trawalter, S., \& Shelton, J. N. (2003). An fMRI investigation of the impact of interracial contact on executive function. Nature Neuroscience, 6(12), 1323-1328.

Ritsher, J. B., Otilingam, P. G., \& Grajales, M. (2003). Internalized stigma of mental illness: Psychometric properties of a new measure. Psychiatry Research, 121(1), 31-49.

Schiller, D., Freeman, J. B., Mitchell, J. P., Uleman, J. S., \& Phelps, E. A. (2009). A neural mechanism of first impressions. Nature Neuroscience, 12(4), 508-514.

Teachman, B. A., Wilson, J. G., \& Komarovskaya, I. (2006). Implicit and explicit stigma of mental illness in diagnosed and healthy samples. Journal of Social and Clinical Psychology, 25(1), 75-95.

Uleman, J. S., Newman, L. S., \& Moskowitz, G. B. (1996). People as flexible interpreters: Evidence and issues from spontaneous trait inference. Advances in experimental social psychology, 28, 211279.

Van Overwalle, F. (2009). Social cognition and the brain: A meta-analysis. Human Brain Mapping, 30(3), 829-858.

Viki, G., Osgood, D., \& Phillips, S. (2013). Dehumanization and selfreported proclivity to torture prisoners of war. Journal of Experimental Social Psychology, 49(3), 325-328.

Wakabayashi, A., Baron-Cohen, S., Wheelwright, S., Goldenfeld, N., Delaney, J., Fine, D., ... \& Weil, L. (2006). Development of short forms of the Empathy Quotient (EQ-Short) and the Systemizing Quotient (SQ-Short). Personality and Individual Differences, 41(5), 929-940. 